\title{
The impact of PLS-SEM training on faculty staff intention to use PLS software in a public university in Ghana
}

\author{
Simon Gyasi Nimako ${ }^{1,}$, Francis Bondinuba Kwesi ${ }^{2}$, Emmanuel Kofi Owusu ${ }^{3}$ \\ ${ }^{1}$ Department of Management Studies Education, University of Education, Winneba, Box 1277, Kumasi Campus, Ghana, Accra Institute of \\ Technology, Accra, Ghana \\ ${ }^{2}$ Kumasi Polytechnic, P.O Box 854, Kumasi-Ghana, School of the Built Environment Institute for Housing and Urban Research (IHURER), \\ Heriot-Watt University, Edinburgh, UK \\ ${ }^{3}$ Department of Accounting Studies Education, University of Education, Winneba, Box 1277, Kumasi Campus, Ghana
}

\section{Email address:}

sim.ekomerce@gmail.com (S. G. Nimako), bondinuba@kpoly.edu.gh (F. K. Bondinuba), owusuekofi@gmail.com (E. K. Owusu)

\section{To cite this article:}

Simon Gyasi Nimako, Francis Bondinuba Kwesi, and Emmanuel Kofi Owusu. The Impact of PLS-SEM Training on Faculty Staff Intention to Use PLS Software in a public university in Ghana. International Journal of Business and Economics Research.

Vol. 3, No. 2, 2014, pp. 42-49. doi: 10.11648/j.ijber.20140302.11

\begin{abstract}
The paper empirically assesses the impact of Partial Least Squares Structural Equation Modelling (PLS-SEM) training on academic staff's intentions to adopt PLS-SEM software in their future research work. Our original contribution to knowledge is the application of the Technology Adoption Model (TAM) to study faculty intention to adopt SEM data analysis software in an under-researched context of developing country Higher Institution of Learning (HIL). Building upon the TAM, we developed a research model that conceptualises PLS-SEM training as an external variable that affects the technology adoption process. The research model was tested using data from 34 faculty members who fully participated in a PLS-SEM training workshop at the College of Technology Education, Kumasi (COLTEK) of University of Education, Winneba. The data was analysed using SmartPLS 2.0 for PLS-SEM analysis. The findings indicate that PLS-SEM training has a positive impact on faculty members' intentions to use the PLS-SEM software in future research. Moreover, the findings confirm the applicability and efficacy of the TAM framework that it can predict about $86 \%$ of faculty members' intention to adopt data analysis software. This paper is one of the initial studies into the adoption of SEM data analysis software by the research community in developing countries HIL context. Despite its limitations, the paper offers important theoretical and managerial contributions. It contributes to the literature in the area of adoption of SEM data analysis software in the information systems literature.
\end{abstract}

Keywords: Developing Countries, Partial Least Squares, Data Analysis Training, Structural Equation Modelling, Technology Adoption

\section{Introduction}

Research is among the three services - teaching, research and community service - that universities provide. As a result, universities make efforts to equip its academic and non-academic staff to develop their research expertise. One way to develop staff confidence and research expertise is through research training and development [1]. Training in the form of research workshops and seminars provides opportunity for staff to interact with experts in the field of research and gain hands-on-experience in academic writing [1]. Specifically, one area that requires hands-on-training workshops for developing faculty researchers' academic writing competency is the knowledge and use of data analysis software packages in quantitative research. The use of data analysis software such as SPSS, STATA, AMOS, LISREL and SmartPLS has become extremely necessary in our global education and research due to its higher power, speed and level of complexity in computational analysis over the human manual systems. Consequently, hands-on training in the use of data analysis software for researchers has gained considerable attention in many HIL [2]. Therefore, university faculty adoption of data analysis software packages can be enhanced through effective training workshops.

It is against this background that the University of Education, Winneba (UEW), organised a one-week training workshop on Partial Least Squares (PLS) for Structural 
Equation Modelling (SEM) for its faculty members, both academic and non-academic in 2013. UEW is the only public university in Ghana that is statutorily mandated to provide teacher education for all level of education in Ghana. Currently UEW operates through its four campuses in Winneba, Kumasi, Asante-Mampong and Ajumako.

PLS-SEM is a second generation data analysis methodology for performing SEM based on Partial Least Square algorithm, and it is increasingly gaining widespread acceptance and use among academics, researchers and professionals in many disciplines [3-5]. By providing faculty and other senior members training in PLS-SEM, the University was implementing strategies in its strategic plan to provide institutional resource and support for developing its staff research expertise in order to catch up with current global demand for evidenced-based scholarly papers.

The study is necessary because it is important for management of UEW to be empirically informed regarding the extent to which the PLS-SEM training influenced staff's intentions to use PLS software and/or methodology. This is a strategic means of providing feedback for management strategy and policy towards development of its faculty research expertise to enable the faculty fit into the current trend of international research paradigms that incorporate rigorous data analysis methodologies.

Moreover, while technology adoption models such as Technology Acceptance Model (TAM) developed by [6] has been applied in many specific research contexts [7-8], very limited attention has been given to research in the adoption of data analysis software by academic staff in developing countries HIL. In particular, since no study, as far as the researchers know, has been conducted to apply the TAM to the specific context of data analysis software adoption among university faculty members, this paper will extend the application of the TAM to data analysis software adoption by faculty members, in HIL in a developing country's context. This will further our theoretical understanding of the extent to which technology adoption factors such as individual's perceived usefulness, perceived ease of use and attitude towards the technology in question can be affected by external facilitating conditions such as training, which has received considerably little attention in the technology adoption literature, especially in developing countries contexts. In view of the above, this paper seeks to assess the impact of PLS-SEM training on academic staff's intentions to adopt PLS-SEM software in their future research work.

Specifically, the paper is guided by the following objectives:

1. To determine the extent to which PLS-SEM training influences academic staff's perceived usefulness, perceived ease of use, attitude and intention to use SEM-PLS software in research.

2. To assess the extent to which the application of TAM is capable of explaining staff intentions to adopt PLS-SEM data analysis software in developing countries HIL such as UEW.

\section{Theoretical Background and Hypothesis}

\subsection{PLS-SEM}

The need for testing cause-and-effect relationships involving many dependent and independent variables simultaneously has led to a growing interest in SEM methodologies and their attendant software development [3, 31]. Two SEM methodologies that dominate the literature are Covariant-based SEM (CB-SEM) and PLS-SEM. PLS-SEM is a component-based causal modelling approach aimed at maximizing the explained variance of the dependent latent constructs [5]. Contrary to CB-SEM methodology, PLS-SEM is preferred for its soft non-parametric distributional assumptions, ability to estimate relationships between latent variables with both formative and reflective indicators, ability to handle complex models and availability of varieties of software for PLS-SEM modelling [3-5].

Some of the software developed for PLS-SEM include SmartPLS, PLS-Graph, WarpPLs and Visual PLS [9]. PLS-SEM has gained widespread acceptance in many academic disciplines [3, 5, 10]. However, some of its limitations are that PLS-SEM is not as yet capable of handling multi-group analysis of more than two groups and its does produce model goodness-of-fit indices as in CB-SEM.

Despite the growing number of studies on PLS-SEM, relatively few researchers in developing countries HIL appear to be familiar with its procedures and use in quantitative research. Given its advantages and growing acceptance in the academic and professional cycles [5], it becomes important for researchers in HIL in developing countries like UEW in Ghana to provide adequate training in PLS-SEM to equip faculty members with the requisite knowledge and skills in PLS-SEM methodology and software to enhance their research competencies.

\subsection{The Technology Acceptance Model (TAM)}

In the information system literature, several models have been developed to help explain the factors that influence user adoption and use of technology, such as computer and information systems software. One such model is the Technology Acceptance Model (TAM) developed by [6]. While there have been several attempts to extend the TAM, its most basic constructs are still relevant in explaining user acceptance and use of technology in many contexts.

The TAM was developed to explain the factors that influence individual's adoption and acceptance of a technology. It provides useful foundation for understanding the factors that drive technology acceptance, and has therefore been one of the most widely adopted theoretical framework in understanding user acceptance of technology [7]. The TAM (see Fig. 1) came from [11]. According to the TAM, perceived usefulness and perceived ease of use of the technology in question are hypothesized to be fundamental 
determinants of user acceptance. The TAM posits that users' actual or behavioural acceptance is principally determined by behavioural intentions to use the technology in question. Behavioural intentions are in turn influenced by the user's attitude towards technology.

Reference [6] maintain that perceived usefulness and perceived ease of use are beliefs that lead to favourable attitudes and intentions to accept and use technology, which in turn affects acceptance and use or adoption of the technology in question. Despite the fact that the TAM has been criticized of having attracted a lot of modifications and extensions, suggesting that the model is inadequate to explain technology acceptance in many research context [7], it continues to be empirically proven for its ability to predict about $40 \%$ of a system use [8, 12-14], and has received praise for its parsimony and predictive powers over the years. The TAM therefore, could become an important reference theory in understanding PLS-SEM software adoption by faculty members in HIL.

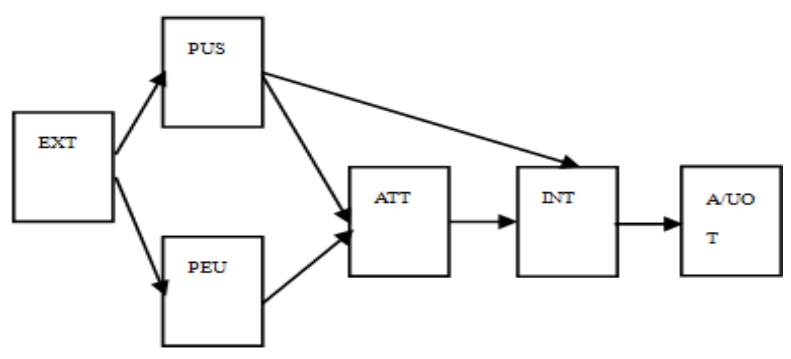

Fig 1. Technology Acceptance Model. Adapted from [6].

Note: INT - Intention to Use Technology, ATT- Computer Attitude, PEU Perceived Ease of Use, PUS - Perceived Usefulness, EXT - External Variables, A/UOT - Acceptance/use of technology

\subsection{Research Model and Hypothesis Development}

The conceptual framework for this study adopts the TAM as its theoretical foundation. It conceptualises PLS-SEM training as part of the external variables that also acts as facilitating condition that can positively affect perceived ease of use (PEU), perceived usefulness (PUS) and attitude towards the use of PLS-SEM software (ATT) by academic staff.

Training is generally used to develop individual's skills, attitudes, abilities, knowledge and competencies in organisations in order to function effectively on a given job or area of responsibility [15]. Training in PLS-SEM for beginners basically involves providing trainees with basic knowledge and skills in understanding and use of PLS-SEM methodology and software. Such training is expected to cover areas such as the overview and underlying assumptions of PLS-SEM methodology as well as its usefulness and procedures, its softwares and limitations in research.

Therefore, in order to influence staff intentions to use PLS-SEM software and methodology in their future work, training in the use of PLS-SEM software becomes one of the key external variable that management of HIL can use. Specifically, it is expected that based on the knowledge and skills gained from PLS-SEM training workshops, participants would develop positive attitude towards the use of PLS-SEM software, gain more understanding of its usefulness, and be able to improve their perception of the ease of use of PLS-SEM software as proposed in the TAM. Thus, in the context of the TAM framework, PLS-SEM training could be described as an external variable that could affect the technology adoption process directly and indirectly.

The role of PLS-SEM training in technology adoption process, especially data analysis software adoption, has received relatively little empirical testing in general and in developing countries HIL contexts in particular. Moreover, though the TAM and its hypothesized relationships among perceived usefulness, perceived ease of use and attitude have received strong theoretical support in some information systems context [7, 8, 12-14], there is the need to extend the model to the context of adoption of data analysis software, which is under-researched and a relatively new area. Moreover, there is evidence of the positive influence of external variables in the technology adoption process in the extant literature in general [14-17]. Therefore, the study extends the basic TAM framework to the adoption of data analysis software by faculty members in HIL in developing countries such as Ghana, and assesses the impact of PLS-SEM training on the decision to adopt the technology. Based on the above discussion, the following hypotheses are proposed:

H1: PLS-SEM training will have significantly positive influence on perceived usefulness of PLS-SEM software for research work.

H2: PLS-SEM training will have significantly positive influence on perceived ease of use of PLS-SEM software for research work.

H3: PLS-SEM training will have significantly positive influence on attitude towards using PLS-SEM software for research work.

H4: PLS-SEM training will have significantly positive influence on intentions to use PLS-SEM software for research work.

H5: Perceived usefulness of PLS-SEM software will have significantly positive influence on attitude towards the use of PLS-SEM software for research work.

H6: Perceived usefulness of PLS-SEM software will have significantly positive influence on intentions to use PLS-SEM software for research work.

H7: Perceived ease of using PLS-SEM software will have significantly negative influence on attitude towards the use of PLS-SEM software for research work.

H8: Attitude towards using PLS-SEM software will have significantly positive influence on intentions to use PLS-SEM software for research work.

\section{Methodology}

The population consisted of participants of a five-day PLS-SEM training workshop organised by the University of Education, Winneba for all senior members at the College of Technology Education, Kumasi Campus (COLTEK) from 
3rd to 7th June 2013. The training sessions involved discussion of conceptual knowledge in PLS-SEM,

demonstrations by a facilitator, and hands-on practical work by participants using SmartPls 2.0 [18].

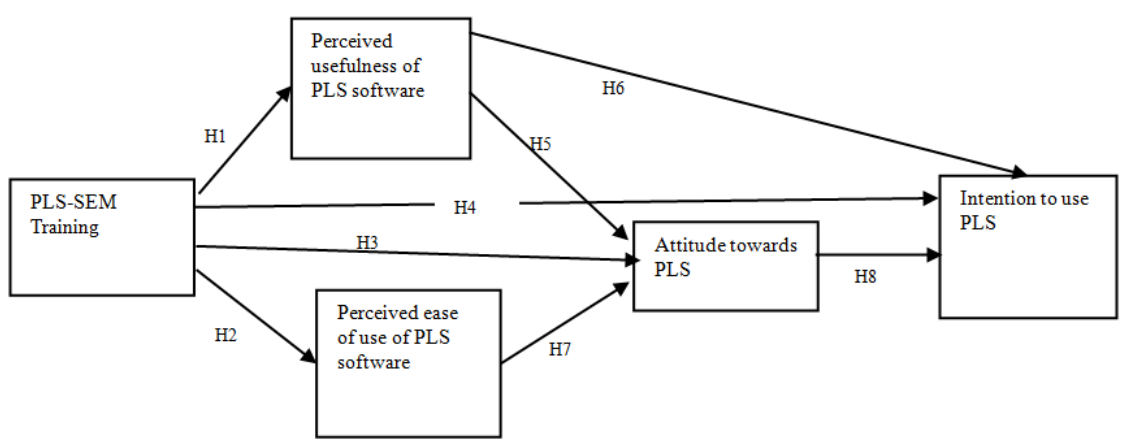

Note: PLS-SEM - Partial Least Squares Structural Equation Modelling

Figure 2. Research model and hypothesized relationships.

Participants who completed the training workshop till the fifth day constituted the sample for the study; they were about 40 in number.

A questionnaire was developed to collect data from participants regarding their intentions to use PLS-SEM in future research work. It had two sections; section A contained 16 items for measuring the research constructs and section B had background data such as gender, age, department, staff status, publishing experience and study area. The questionnaire was distributed to participants to complete them and return at the end of the closing ceremony. In all 34 questionnaire were retrieved representing 85\% response rate.

\section{Data Analysis and Results}

Data was analysed using SmartPLS 2.0 [18] to perform Partial Least Squares Structural Equation Modelling (PLS-SEM) to test the hypothesized relationships among the constructs in the proposed model depicted (see Fig. 1). PLS-SEM was deemed most appropriate because of the predictive focus of the study [3]

For sample size considerations in PLS-SEM, according to reference [5, p. 144], as a common rule of thumb for appropriate sample size for testing PLS-SEM models is the rule of ten, which suggests ten times the largest number of structural paths directed at a particular latent construct in the structural model. In this study, the highest number of indicators for a construct was three and three structural paths at a time. Hence three multiplied by ten gives 30 cases; thus, our sample 34 respondents could be described as adequate. The SmartPLS 2.0 software was set to 500 bootstrap samples for the estimation of significance of the t-values. Generally, the data analysis followed [19]'s two-step approach: estimation of the measurement model before the structural model.

\subsection{Respondents' Background Data}

In terms of gender, majority were males $(91 \%)$ and few females $(9 \%), 38 \%$ of them were below 35 years of age,
$19 \%$ were between 36 and 55 years and $2 \%$ were above 56 years and above. $44 \%$ were from the business faculty, $29 \%$ were in technology faculty, $25 \%$ were from other faculties. $82 \%$ were academic staff and $18 \%$ were non-academic staff. In terms of research experience, $65 \%$ of them had published less than five research papers, $13 \%$ had published from 5 to 15 research papers while $20 \%$ had not yet published any research paper. One person $(2.9 \%)$ had used SEM in previous work, the rest had not yet used SEM in research. $29 \%$ of the respondents favoured quantitative research philosophy and about $71 \%$ favoured qualitative approach.

\subsection{Measurement Model Reliability and Validity}

Table 1. Cross Loadings for Convergent validity

\begin{tabular}{llllll}
\hline $\begin{array}{l}\text { Construct/ } \\
\text { Indicators }\end{array}$ & ATT & INT & PEU & PUS & TR \\
\hline ATT1 & $\mathbf{0 . 9 2 5}$ & 0.819 & 0.712 & 0.716 & 0.773 \\
ATT2 & $\mathbf{0 . 8 7 8}$ & 0.733 & 0.585 & 0.681 & 0.670 \\
ATT3 & $\mathbf{0 . 8 7 7}$ & 0.801 & 0.709 & 0.754 & 0.768 \\
INT1 & 0.704 & $\mathbf{0 . 9 4 1}$ & 0.692 & 0.743 & 0.799 \\
INT2 & 0.705 & $\mathbf{0 . 9 6 7}$ & 0.700 & 0.741 & 0.721 \\
INT3 & 0.760 & $\mathbf{0 . 8 8 1}$ & 0.539 & 0.713 & 0.812 \\
PEU1 & 0.705 & 0.586 & $\mathbf{0 . 8 3 3}$ & 0.592 & 0.732 \\
PEU2 & 0.589 & 0.537 & $\mathbf{0 . 7 7 0}$ & 0.552 & 0.458 \\
PEU3 & 0.453 & 0.513 & $\mathbf{0 . 7 5 6}$ & 0.466 & 0.525 \\
PUS1 & 0.616 & 0.563 & 0.602 & $\mathbf{0 . 7 7 9}$ & 0.635 \\
PUS2 & 0.652 & 0.666 & 0.587 & $\mathbf{0 . 8 0 4}$ & 0.663 \\
PUS3 & 0.832 & 0.821 & 0.515 & $\mathbf{0 . 8 6 6}$ & 0.783 \\
TR1 & 0.739 & 0.690 & 0.690 & 0.671 & $\mathbf{0 . 8 4 2}$ \\
TR2 & 0.756 & 0.841 & 0.622 & 0.709 & $\mathbf{0 . 8 7 1}$ \\
TR3 & 0.779 & 0.746 & 0.630 & 0.715 & $\mathbf{0 . 9 0 4}$ \\
\hline
\end{tabular}

Notes: Item loadings are in bold, ATT - attitude toward PLS-SEM software, INT - Intention to use PLS-SEM software, PEW - Perceived ease of use of PLS-SEM software, PUS - Perceived usefulness of PLS-SEM software, TR - PLS-SEM Training

Construct reliability measures the extent of internal consistency of measures used, and it is assessed through 
Cronbach's alpha with the acceptable level of 0.7 for basic research and also through composite reliability, with acceptable value of 0.70 [20].

From Table 2, all of the constructs have higher Cronbach alphas above 0.70 , indicating that these multiple measures are highly reliable for the measurement of each construct. Construct validity assesses the degree to which a measurement represents and logically connects the observed phenomenon to the construct through the fundamental theory [21]. It is assessed through convergent validity and discriminant validity [20].

Table 2. Construct Reliability and discriminant validity

\begin{tabular}{ccccccccc}
\hline & ATT & INT & PEU & PUS & TR & AVE & CR & CA \\
\hline ATT & $\mathbf{0 . 8 9}$ & & & & & 0.80 & 0.92 & 0.87 \\
INT & 0.81 & $\mathbf{0 . 9 3}$ & & & & 0.87 & 0.95 & 0.92 \\
PEU & 0.75 & 0.70 & $\mathbf{0 . 7 9}$ & & & 0.62 & 0.83 & 0.70 \\
PUS & 0.77 & 0.75 & 0.69 & $\mathbf{0 . 8 2}$ & & 0.67 & 0.86 & 0.75 \\
TR & 0.87 & 0.81 & 0.74 & 0.76 & $\mathbf{0 . 8 7}$ & 0.76 & 0.91 & 0.84 \\
\hline
\end{tabular}

Notes: AVEs are in the diagonal; correlations are below the diagonal; AVE-Average variance extracted, CR- Composite reliability, CA Cronbach's alpha

Convergent validity can be assessed through construct factor (item) loadings (or cross loadings in PLS), average variance extracted (AVEs) that should have minimum loading of 0.5, and composite reliability (CR) with acceptable minimum of 0.70 [19-21]. From Table 1, the factor loadings of items to their respective constructs are stronger than they load on other constructs providing evidence in support of the convergent validity of the derived measures. Moreover, the $\mathrm{CR}$ values for all constructs range from 0.830 to 0.951 exceeding the acceptable requirement of 0.70 confirming convergent validity of the measurement (outer) model.

Discriminant validity was considered adequate since the AVEs are greater than their respective inter-construct correlations as is in Table 2 [21]. Given that construct reliability and validity conditions of the measurement model are acceptable, we proceed to assess the psychometric properties of the structural model.

\subsection{Results of Structural Model}

In PLS-SEM, structural models' validity are assessed through the strength of regression weights, t-values, $p$-values for significance of t-statistics, as well as effect sizes of independent variables on the dependent variables. The results in Table 3 shows that, all the hypotheses were supported by the data. Specifically, it indicates that PLS-SEM training have significantly positive effect on intention to adopt, attitude, perceived ease of use and perceived usefulness of PLS-SEM software, providing support for H1 to H4. Among them, PLS-SEM training made the strongest impact on perceived ease of use and perceived usefulness than on the others.
Moreover, perceived usefulness significantly influenced attitude and intentions, exerting greater effect on attitude than on intention. These results provide support for H5 and H6 respectively. Finally, perceived ease of use significantly influenced attitude and attitude influenced intentions to adopt, providing evidence in support of $\mathrm{H} 7$ and $\mathrm{H} 8$ respectively.

\subsection{Results of Predictive Power and Effect Sizes}

The R-Square measures the predictive power of the structural model in PLS analysis. The predictive power of each independent variable to their respective dependent variables were conducted by eliminating each of the dependent variable in question, one at a time in an iterative process. The effect size is then estimated as:

$$
\frac{R^{2} \text { included }-R^{2} \text { excluded }}{1-R^{2} \text { included }}
$$

The effect size of each of the independent variables is presented in Table 4. Reference [22] provides the following guidelines for interpreting effect sizes: Less than $0.02-$ no effect, Small - 0.02, medium -0.15 , large -0.35 .

From Table 4 , the model as a whole predicts about $86 \%$ of intention of staff to adopt PLS-SEM software, indicating a very large effect size. This implies that all the dependent variables (ATT, PEU, PUS and TR) collectively predict intentions to adopt strongly. The predictive power of the other dependent variables, ATT $\left(\mathrm{R}^{2}=0.829\right)$, PEU $\left(\mathrm{R}^{2}=\right.$ $0.554)$ and PUS $\left(R^{2}=0.732\right)$ also suggests large effect sizes. Overall, attitude made the greatest impact on intention to adopt with a large effect size $\left(f^{2}=0.784\right)$, followed by perceived usefulness with a medium effect size $\left(f^{2}=0.194\right)$, while PLS-SEM training made the least impact with a small effect size on intention to adopt $\left(f^{2}=0.115\right)$.

However, in the full model PLS-SEM training made large effects on PEU $(\mathrm{R} 2=0.554)$ and PUS $(\mathrm{R} 2=0.732)$ as the only predictor of the two. Also, the contribution of PLS-SEM training to ATT is a medium effect size (f2 = 0.152 ). Finally, PEU and PUS had small ( $\mathrm{f} 2=0.099$ ) and (f2 $=0.287)$ medium effect sizes on ATT respectively.

\section{Discussion and Implications}

The In this research, the overarching purpose was to assess the impact of PLS-SEM training on academic staff's intentions to adopt PLS-SEM software in their future research work. Using data from participants of the training workshop, the results show that the PLS-SEM training had significantly positive and direct effect on respondents' intention to adopt PLS-SEM software in future research. PLS-SEM training also had significantly positive effect perceived usefulness, perceived ease of use and attitude towards the use of PLS-SEM software. Based on the results the paper makes the following theoretical and managerial contributions. 
Table 3. Results of hypothesis testing

\begin{tabular}{ccccccc}
\hline Hypothesis & Path & Regression weight & Standard Error & T Statistics & p-value & Remarks \\
\hline H1 & TR - > PUS & 0.856 & 0.009 & 97.775 & $0.000^{* * *}$ & Supported \\
H2 & TR -> PEU & 0.741 & 0.019 & 38.611 & $0.000^{* * *}$ & Supported \\
H3 & TR -> ATT & 0.361 & 0.035 & 10.301 & $0.000^{* * *}$ & Supported \\
H4 & TR - INT & 0.295 & 0.033 & 8.873 & $0.000^{* * *}$ & Supported \\
H5 & PUS - ATT & 0.426 & 0.037 & 11.443 & $0.000^{* * *}$ & Supported \\
H6 & PUS -> INT & 0.123 & 0.022 & 5.614 & $0.000^{* * *}$ & Supported \\
H7 & PEU - ATT & 0.193 & 0.022 & 8.777 & $0.000^{* * *}$ & Supported \\
H8 & ATT -> INT & 0.586 & 0.036 & 16.390 & $0.000^{* * *}$ & Supported \\
\hline
\end{tabular}

Note: ATT - attitude toward PLS-SEM software, INT - Intention to use PLS-SEM software, PEU - Perceived ease of use of PLS-SEM software, PUS Perceived usefulness of PLS-SEM software, TR - PLS-SEM Training

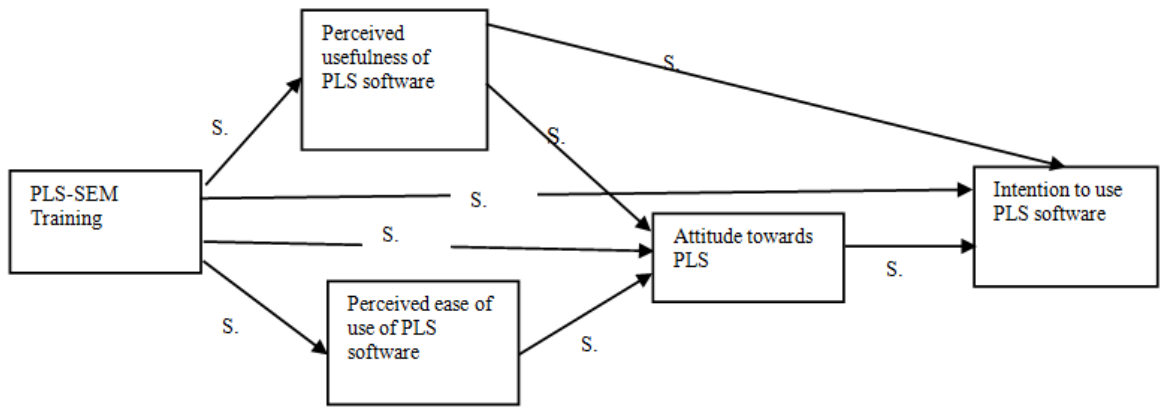

Note: S. - significant relationships

Figure 3. Results of hypothesis testing

Table 4. Effect size analysis

\begin{tabular}{|c|c|c|c|c|c|}
\hline Models & Dependent variables & $\mathbf{R}^{2}$ included & $\mathbf{R}^{2}$ excluded & $\mathbf{f}^{2}$ & Remarks \\
\hline \multirow[t]{4}{*}{ Full model } & INT & 0.861 & N/A & N/A & Large effect \\
\hline & PUS & 0.732 & N/A & N/A & Large effect \\
\hline & ATT & 0.829 & N/A & N/A & Large effect \\
\hline & PEU & 0.554 & N/A & N/A & Large effect \\
\hline Model without PEU & ATT & 0.829 & 0.812 & 0.099 & Small effect \\
\hline \multirow[t]{2}{*}{ Model without PUS } & ATT & 0.829 & 0.780 & 0.287 & Medium effect \\
\hline & INT & 0.861 & 0.834 & 0.194 & Medium effect \\
\hline Model without ATT & INT & 0.861 & 0.752 & 0.784 & Large effect \\
\hline \multirow[t]{2}{*}{ Model without TR } & INT & 0.861 & 0.845 & 0.115 & Small effect \\
\hline & ATT & 0.829 & 0.803 & 0.152 & Medium effect \\
\hline
\end{tabular}

Notes: $* * *$ significant at $0.001, *$ significant at 0.05 , effective size: 0 - none, $0.02-$ small, 0.15 - medium, 0.35 - large [22], N/A - Not applicable

Theoretically, the paper makes three contributions. First, it has contributed to the literature on adoption and use of technology by testing the TAM in developing countries HIL, which is a new and under-researched context for the research problem. The findings confirm that, faculty members in developing countries who are new to SEM methodologies in general and PLS-SEM in particular can be influenced to develop positive attitudes and strong intentions to adopt PLS-SEM methodology and software through effective training in PLS-SEM.

Second, the paper advances our understanding of the external variables outside the individual that act as antecedents to intention to accept and use technology. More specifically, the study has demonstrated that training in the use of the technology in question (i.e. PLS-SEM software) can make a positive and direct effect on intentions to adopt the technology, and can indirectly have a positive and strong influence on adoption intentions through other significant antecedents in the adoption process such as perceived usefulness, perceived ease of use and attitude towards the technology in question.

The reason for the positive and direct relationship between PLS-SEM training and intentions to adopt PLS-SEM software could be explained through self-efficacy. Since people's behaviour is strongly influenced by their self-efficacy, which is the confidence in one's ability to perform a given task [23-24], it follows that PLS-SEM training could increase individual's belief in their ability to use PLS-SEM software in future research. This could in turn positively influence their intention to adopt a given technology [25]. Future research is needed to explore the moderating factors such as individual researcher's research philosophy, demography as well as academic discipline and research specialisations that are likely to influence the relationship between SEM training and intentions to adopt SEM technologies.

Three, apart from the new lights the study sheds, the results also confirm a number of previous studies regarding the 
positive effect of perceived usefulness on attitude [e.g., 6-8, 13], the positive effect of perceived ease of use on attitude [e.g., 13, 14] and the significance of attitude-intention relationship in general and in the context of technology adoption in particular [e.g., 12, 13, 25-27]. It has, therefore, demonstrated the applicability of the TAM framework in predicting individual's intentions to accept and use technology, confirming the claims of the applicability and efficacy of the TAM $[6,7]$. In particular, in the present study, the finding that the TAM strongly predicts about $86 \%$ of adoption in a developing country HIL context. It contributes to the debate on the efficacy of the TAM that the basic TAM model continues to have the ability to predict individual's adoption of technology in spite of the limitations and extensions it has received over the years in the extant literature.

To management and other practitioners, the findings of the present study make two practical contributions. First, management of HIL, especially among faculty members who are new to SEM in many developing countries, can positively influence faculty members' intention to accept and use of second generation SEM data analysis methodologies by supporting them through effective training in PLS-SEM, CB-SEM and other similar methods in research. In order to realise its full potential benefits, such data analysis and research training workshops ought to have both the conceptual, knowledge-based component as well as the hands-on-experience and application component [15, 28].

The findings show that there are strong hopes that developing countries HIL faculty members who are relatively new to the use of SEM statistical software packages can be trained to acquire knowledge and skill for using SEM methodology in future research. Such training should be frequently organised and well-resourced. This will enable staff to effectively contribute to the current research in top tier academic and professional journals that publish rigorous, evidence-based, theory-driven research and empirical work. This will in turn help bridge the perceived global research-divide, especially between developing countries in Africa and their North American and European counterparts, who seem to have dominated, in terms of published papers and editorial positions, most of the top international journals for a long time [29, 30].

Second, the findings of the present study also imply that data analysis and similar research training workshops for researchers who are new to the use of SEM software should focus on three key areas. Thus, the training content should focus on developing positive attitude, greater perceived usefulness and ease of use towards the use of SEM softwares. One way to develop these three areas is for trainers to highlight the rationale and importance of any SEM software, the underlying theoretical foundations, the details of procedures involved, how to overcome fears and difficulties that come with the use of the software as well as providing opportunity for trainees to practice freely under supervision of other training assistants [28].

\section{Limitations and Future Research}

While this study makes important contributions to theory and management of HIL, it is limited in context and in terms of variables captured. The use of participants in a PLS-SEM training at COLTEK, only one of the four campuses of UEW places some limit on the generalizability of the findings. Future research is recommended in attempting to study the impact of data analysis training workshop on participants' intention to adopt technology in other universities elsewhere in the world using larger population. Moreover, there are a number of other external factors that can affect the adoption of the PLS-SEM apart from the training in the use of technology. These factors may include individual researchers' research philosophy, demography as well as academic discipline and research specialisations. Future research should explore the extent to which these other factors can affect individual's technology adoption intentions.

\section{Conclusion}

In this study the effect of PLS-SEM training on academic staff's intention to adoption PLS-SEM software in future research has been assessed. The findings have provided empirical evidence on the effect of external variables such as hands-on-training in the use of a given technology on individual's technology adoption process. It has confirmed the findings of many previous studies on the efficacy of the TAM framework. It has also extended the framework to a new context by demonstrating how constructs in the TAM could help predict faculty members' intention to adopt PLS-SEM software in their future research work in a developing country HIL context. The implications of the findings are important for management in two areas. One is the development of faculty research expertise through effective and periodic training, and the other is that, the training workshop should be hands-on, practical work focusing on influencing perceived usefulness, ease of use and attitude towards the use of the software. The study is limited in terms of generalizability of the findings in HIL in developing countries due to the fact that the sample used consisted of only academic staff of COLTEK, one of the campuses of UEW. Areas for future research have been suggested for future research on the role of SEM training on adoption of SEM software as a means of improving research quality and output of faculty members in HIL in developing countries.

\section{References}

[1] Nimako, S.G., Danso, H. and Donkor, F. (2013). Using Research Workshop to Assist Senior Members Develop Competence in Academic Writing in a Public University. Journal of Studies in Social Sciences, 5 (2), 301-327.

[2] Lee, M. K. O., Cheung, C. M. K. and Chen, Z. (2005). Acceptance of Internet-based learning medium: The role of extrinsic and intrinsic motivation. Information \& Management, 42, pp. 1095-1104. 
[3] Chin, W. (2010) How to write up and report PLS analyses. In: EspositoVinzi V, Chin W.W, Henseler J, Wang H (eds) Handbook of partial least squares: concepts, methods and applications. Springer Heidelberg, pp. $655-690$.

[4] Esposito Vinzi, V., Chin, W. W., Henseler, J. and Wang, H. (2010) Handbook of partial least squares: Concepts, methods and applications, pp. 2 - 35.

[5] Hair, J. F., Ringle, C. M., and Sarstedt, M. (2011) PLS-SEM: Indeed a Silver Bullet, Journal of Marketing Theory and Practice 19 (2), pp. 139-151.

[6] Davis, F. D., Bagozzi, R. P. and Warshaw, P. R. (1989) User acceptance of computer technology: A comparison of two theoretical models. Management Science, 35(8), 982- 1003.

[7] Bagozzi R.P., (2007) The legacy of the technology acceptance model and a proposal for a paradigm shift, Journal of the Association for Information Systems, 8(4): pp. 244-254.

[8] Legris, P., Ingham, J. and Collerette, P. (2004) Why do people use information technology? A critical review of the technology acceptance model. Information \& Management, $40,191-204$.

[9] Temme, D., Kreis, H. and Hildebrandt, L. (2010) A comparison of current PLS path modelling software: features, ease-of-use, and performance. In Handbook of Partial Least Squares (pp. 737-756). Springer Berlin Heidelberg.

[10] Ringle, C., Sarstedt M. and Straub, D. (2012) A critical look at the use of pls-sem in mis quarterly. MISQ 36(1): iii-xiv

[11] Ajzen, I. and Fishbein, M. (1980), Understanding attitudes and predicting social behavior, Englewood Cliffs, NJ: Prentice Hall.

[12] Lin, J. C., and Lu, H. (2000) Towards an understanding of the behavioral intention to use a Web Site. International Journal of Information Management, 20, pp. 197-208.

[13] Park, S. Y. (2009). An Analysis of the Technology Acceptance Model in Understanding University Students' Behavioral Intention to Use e-Learning. Educational Technology \& Society, 12 (3), 150-162.

[14] Pituch, K.A, \& Lee, Y.-K. (2006). The influence of system characteristics on e-learning use. Computers Education, 47, 222-244.

[15] Saks, A. M., Tamkin, P. and Lewis, P. (2011) Management training and development. International Journal of Training and Development, 15(3), 179-183.

[16] Liu, S., Liao, H. and Peng, C. (2005) Applying the technology acceptance model and flow theory to online e-learning users' acceptance behavior. Issues in Information Systems, 6(2), pp. 175-181.

[17] Saadé, R. G. (2003). Web-based education information system for enhanced learning, EISL: Student assessment.
Journal of Information Technology Education, 2, 267-277.

[18] Ringle, C.M., Wende, S. and Will, S. (2005). SmartPLS 2.0 (M3) Beta. Hamburg, http://www.smartpls.de.

[19] Anderson, J.C. and Gerbing, D.W. (1988) Structural equation modelling in practice: A review and recommended two-step approach, Psychol. Bull, 103 (3), pp. 411-423.

[20] Hair, J. F., Black, W. C., Babin, B. J. and Anderson, R. E. (2010) Multivariate Data Analysis, Englewood Cliffs, NJ: Prentice Hall.

[21] Fornell, C. and Larcker, D. F. (1981) Evaluating structural equation models with unobservable variables and measurement error. Journal of Marketing Research, 18, $39-50$

[22] Cohen, J. (1988) Statistical Power Analysis for the Behavioral Sciences. HillsDale, NJ: Lawrence Erlbaum.

[23] Ajzen, I. (1991) The theory of planned behavior. Organizational Behavior and Human Decision Processes, 50, pp.179-211.

[24] Bandura, A. (1994) Self-efficacy. In V. S. Ramachaudran (Ed.), Encyclopedia of human behaviour (pp. 71-81). New York, NY: Academic Press.

[25] Grandon, E., Alshare, O., \& Kwan, O. (2005). Factors influencing student intention to adopt online classes: A cross-cultural study. Journal of Computing Sciences in Colleges, 20(4), 46-56.

[26] Ajzen, I. and Fishbein, M. (2000) Attitudes and the Attitude Behavior Relation: Reasoned and Automatic Processes, European Review of Social Psychology 11 (1), pp. 1-33.

[27] Ajzen, I. and Fishbein, M. (2005) The Influence of Attitudes on Behavior, in The Handbook of Attitudes, D. Albarracín, B. T. Johnson, and M. P. Zanna (eds.), Mahwah, NJ: Erlbaum, pp. 173-221.

[28] Reisdorph, N., Stearman, R., Kechris, K., Phang, T. L., Reisdorph, R., Prenni, J., ... \& Geraci, M. (2013). Hands-on Workshops as An Effective Means of Learning Advanced Technologies Including Genomics, Proteomics and Bioinformatics. Genomics, proteomics \& bioinformatics, 11(6), 368-377.

[29] Svensson, G. (2005) Ethnocentricity in top marketing journals, Marketing Intelligence \& Planning, Vol. 23 Iss: 5, pp. $422-434$

[30] Svensson, G., Tronvoll, B., Helgesson, T. and Slåtten, T. (2009) The 'geographical affiliations' in 'top' research journals of general marketing. Australasian Marketing $\begin{array}{llll}\text { Journal (AMJ), } & 17(3), & 154-159 .\end{array}$ DOI.10.1108/02634500510612618

[31] Jöreskog, K.G. and Sörbom, D. (1986) LISREL VI: Analysis of linear structural relationships by maximum likelihood and least squares methods. Scientific Software, Mooresville, IN 